\title{
Vrai procès et faux débats : perspectives critiques sur les argumentaires de légitimation des entreprises de coercition para-privées
}

\section{Christian Olsson}

\section{(2) OpenEdition \\ Journals}

Édition électronique

URL : http://journals.openedition.org/conflits/975

DOI : $10.4000 /$ conflits. 975

ISSN : $1777-5345$

Éditeur :

CCLS - Centre d'études sur les conflits lilberté et sécurité, L'Harmattan

Édition imprimée

Date de publication : 1 décembre 2003

Pagination : 11-48

ISBN : 2-7475-6065-1

ISSN : 1157-996X

Référence électronique

Christian Olsson, «Vrai procès et faux débats : perspectives critiques sur les argumentaires de

légitimation des entreprises de coercition para-privées », Cultures \& Conflits [En ligne], 52 | hiver 2003,

mis en ligne le 03 juillet 2004, consulté le 30 mars 2021. URL : http://journals.openedition.org/conflits/ 975 ; DOI : https://doi.org/10.4000/conflits.975

Ce document a été généré automatiquement le 30 mars 2021.

Creative Commons License 


\title{
Vrai procès et faux débats : perspectives critiques sur les argumentaires de légitimation des entreprises de coercition para- privées
}

\author{
Christian Olsson
}

Dans un contexte dans lequel les mercenaires individuels font encore couler beaucoup d'encre dans les quotidiens à chaque fois que d'anciens militaires français, russes ou ukrainiens veulent s'impliquer en RDC, Madagascar ou en Côte d'Ivoire, ce sont néanmoins les «entreprises militaires privées" (private military companies) qui représentent l'essentiel des soldats professionnels opérant dans le secteur privé aujourd'hui. Ils constitueraient le deuxième contingent le plus important de la coalition internationale en Irak après celui de l'armée américaine, mais avant celui de l'armée britannique ${ }^{1}$. Au Kosovo, en 1998, la totalité du contingent américain de la mission internationale d'observation du Kosovo Monitoring Group fut sous-traité à l'entreprise DynCorps. Pourtant, contrairement aux mercenaires individuels, ces entreprises (ainsi que leur personnel) ne semblent pas faire l'objet d'une réprobation quasi-unanime, et ainsi le fondement éthique de leurs activités est aujourd'hui sujet à débat. Cet article s'intéresse à ce dernier débat, ou plus exactement aux discours des défenseurs de l'option privée, en essayant de montrer quels en sont les ressorts et les travers. Cependant bien loin de se réduire à une simple analyse de discours, il a aussi la prétention de confronter ces argumentaires de légitimation aux pratiques sociales des entreprises de coercition militaire.

Les entreprises de coercition para-privées en question : la structure d'un faux débat Du point de vue normatif, le débat médiatique, politique, mais aussi académique sur les « entreprises militaires privées » est structuré autour de la question de la légitimité du monopole de la violence légitime, supposé définir l'Etat, dans la gestion des problèmes 
sécuritaires contemporains. Cette question est alors posée par rapport aux solutions proposées par des acteurs privés sur le marché. Cette façon de poser les termes du débat dessine deux grandes perspectives qui, tout en étant antinomiques, permettraient de résumer l'ensemble des postures possibles vis-à-vis de la question de ce "néo-mercenariat » : selon la première, l'efficacité supposée du marché par rapport à celui de l'Etat dans le domaine de la sécurité rendrait légitime le contournement des voies d'interventions militaires classiques (étatiques) en cas de crise internationale ou interne ${ }^{2}$; selon la deuxième, la prééminence dans les enjeux de sécurité des Etats, seuls acteurs responsables et représentatifs, ferait des entrepreneurs militaires privés des acteurs illégitimes ${ }^{3}$. Dans cette dernière perspective, toute intrusion de la part d'acteurs privés dans la sphère de la politique de sécurité constitue un crime de lèsesouveraineté.

Par conséquent, la manière dont le débat est mené aujourd'hui fait que toute critique, autre que circonstancielle, adressée aux entreprises privées de sécurité (inter-)nationale passe forcément par une apologie du construit stato-national. D'ailleurs, il suffit d'évoquer les connotations fortement péjoratives de la notion de «mercenaire " (fait bien perçu par les entreprises de coercition qui rejettent de manière unanime ce qualificatif) pour souligner à quel point cette notion reste entachée du sceau indélébile de non-allégeance et de déloyauté vis-à-vis de l'Etat d'origine. Dès lors, on est soit un tenant de la privatisation de la fonction de sécurité, soit un tenant du monopole absolu de l'Etat sur les moyens de la contrainte militaire et/ou policière. Ce qui est au centre du débat est donc avant tout l'idéalisme statonational et non les entreprises elles-mêmes.

Que cet effet soit intentionnel ou pas, le moins que l'on puisse dire est que cette façon de poser les questions semble biaiser le débat en faveur des tenants des entreprises militaires privées. En effet, ceux-ci peuvent facilement se défendre de subvertir l'ordre juridique international fondé sur la prééminence des Etats, en affirmant que ces entreprises servent les intérêts et les objectifs des Etats et de l'ordre inter-étatique qui représentent l'essentiel de leurs clients. Cela même si ces entreprises constituent de facto une remise en question du monopole public sur la violence légitime dans son acception la plus stricte. Elles peuvent ainsi se prévaloir d'incarner «le meilleur des deux mondes » : l'efficacité présumée du marché et la légitimité présumée du monde inter-étatique. Cet argument est effectivement utilisé par des «praticiens » comme Tim Spicer de Sandline International et Doug Brooks du consortium International Peace Operations Association (IPOA), des analystes comme David Shearer, mais aussi des universitaires comme Christopher Coker, pour balayer d'un revers de main les critiques émises.

Cependant pour peu que l'on ne veuille pas laisser le monopole théorique de la violence légitime aussi monopoliser le débat sur la "privatisation de la violence ", d'autres perspectives s'ouvrent. En effet, pourfendre le mercenariat n'est pas forcément défendre coûte que coûte le monopole de l'Etat sur la violence légitime. Cela parce qu'un tel a priorinormatif resterait prisonnier d'une pensée d'Etat qui néglige les dynamiques propres du phénomène en question. Poser le débat en des termes aussi binaires, c'est forcément tomber dans un des deux écueils qu'il s'agit justement d'éviter lorsque l'on raisonne sur le phénomène des entreprises militaires. Le premier suppose de considérer que ces entreprises sont le révélateur de la fin de la capacité des gouvernements à contrôler les moyens de la contrainte physique ${ }^{4}$. Or cela n'est vrai 
qu'à condition de confondre le concept institutionnel de gouvernement avec celui socio-historique d'Etat ${ }^{5}$ et de réifier les "Etats" au point de considérer qu'il s'agit d'acteurs unitaires dont les frontières d'avec la société, le marché ou le secteur privé sont linéaires et intangibles. La frontière entre le public, relevant de l'«Etat», et le privé, relevant du marché où de la société civile, serait clairement délimitée.

Or, un tel point de vue ne tient pas assez compte de la porosité des frontières entre le public et le privé, des champs transversaux des pratiques de sécurité (qui cassent la distinction public/privé) ainsi que des stratégies d'instrumentalisation réciproque entre acteurs se positionnant dans le secteur privé et acteurs se positionnant dans le secteur public. Il en découle que plutôt que de se contenter de récits sur le déclin des "Etats» (lorsque ce terme est utilisé en lieu et place de "gouvernement»), il faudra faire une sociologie du personnel de ces entreprises, et plus particulièrement de leurs cadres dirigeants afin de mettre en évidence les liens entre le personnel des entreprises privées et les professionnels traditionnels de la sécurité (militaires, gendarmes, police, services de renseignement). En effet, les compagnies militaires privées et la politique internationale de certaines bureaucraties gouvernementales sont souvent difficilement séparables. Ainsi nous parlerons ici d'« entreprises de coercition para-privées " pour souligner le fait que ces firmes s'inscrivent souvent dans des réseaux de personnels qui sont transversales à la simple opposition privé/public et parfois même transnationales ${ }^{6}$. Cette notion permettra également d'éviter le deuxième écueil principal. En effet, certains déduisent des liens étroits existants entre entreprises de sécurité privée et bureaucraties gouvernementales, qu'il ne s'agit que d'organisationsécran, de purs instruments à la solde des «Etats » et de leurs stratégies. Or une telle approche néo-réaliste du phénomène tombe dans la même fiction théorique de $l^{\prime}$ «Etat » unitaire et rationnel au détriment de la mise en évidence de l'autonomie des réseaux de professionnels de la sécurité par rapport aux «hautes sphères » du gouvernement. Les entreprises de coercition para-privées ne relèvent ni du marché pur, ni d'une vision essentialisée de l'Etat.

Nous aborderons dans cet article l'ensemble des entreprises de coercition para-privées faisant usage, dans un but notamment lucratif et sous couvert du label sécuritaire, de technologies de coercition et de surveillance traditionnellement considérées comme militaires. Il ne s'agira donc pas de s'intéresser exclusivement aux entreprises affichant ouvertement leur nature militaire à la fois au travers des moyens employés que des services proposés (ce que traduit le terme anglo-saxon de private military company). Par contre, même si nous traiterons des entreprises de sécurité de type militaire au sens large, ainsi que des « réseaux » de professionnels de la sécurité qui les sous-tendent, les réseaux autonomes dépourvus de toute structure entrepreneuriale ne seront pas abordés ici. Nous centrerons également l'analyse sur les entreprises à vocation internationale au détriment de celles qui se restreignent à n'opérer que sur le territoire national. Ce choix méthodologique découle de notre souci d'étudier les discours de légitimation et les stratégies élaborées de communication en faveur de la privatisation de la fonction de sécurité à l'échelle internationale. Or, celles-ci sont essentiellement le fait des "nouvelles" entreprises militaires à vocation internationale et non des mercenaires engagés à titre individuel.

Du point de vueempirique, l'approche adoptée par la grande majorité des discours sur le «nouveau mercenariat » ou les "entreprises militaires privées », et cela quelle que soit l'appréciation normative du phénomène, consiste à en chercher les causes, généralement en postulant l'existence d'un marché de la sécurité privée dont les seules 
variations de l'offre et de la demande feraient office de variable explicative ${ }^{7}$. Selon cette façon de voir les choses, la multiplication des entreprises de coercition para-privées depuis les années 1990 s'expliquerait par une explosion simultanée de l'offre et de la demande de sécurité privée. L'offre de sécurité privée se serait considérablement accrue avec la fin de la guerre froide et la professionnalisation des armées, accompagnée d'une réduction des effectifs militaires, qui s'en est suivie. En effet, cela aurait libéré une main d'œuvre qualifiée au profit de l'offre privée. Parallèlement, la demande de sécurité aurait crû exponentiellement dans un contexte de démultiplication de crises régionales (le plus souvent expliquées au travers d'une grille de lecture en termes d'«Etats effondrés») à intérêt stratégique limité pour les gouvernements occidentaux. Ces deux seules données suffiraient à rendre compte de l'émergence et de la croissance de l'industrie de la sécurité privée de type militaire. Une telle approche, qui en tant que telle se veut le plus souvent descriptive et explicative, revient pourtant à dépolitiser et à dé-problématiser le fait de la sécurité privée dès lors qu'elle pose l'existence d'un marché de la sécurité privée comme un fait naturel. C'est donc faire peu de cas du fait que ce marché, comme toute institution et tout système de pratiques, est enchâssé dans un système discursif de croyances qui présente le recours à ces entreprises comme légitime.

En amont de l'offre et de la demande, se pose donc la question de la justification et de la légitimation de ces entreprises. Plutôt que d'expliquer la montée en puissance de la sécurité privée par des prétendues lois intemporelles et a-historiques du marché, il convient donc de l'interpréter au travers des systèmes de croyances qui sont sousjacents à l'institutionnalisation progressive d'un marché de la sécurité posant cette offre et cette demande comme allant de soi. En effet, l'interprétation par les forces du marché ne fait que supposer ce qui est vraiment problématique, à savoir l'idée que la sécurité serait un bien comme les autres pouvant être échangé sur un marché. Ainsi, outre l'explication par le néo-libéralisme ambiant ou par l'hypothèse de cultures institutionnelles propices à l'émergence de telles entreprises (hypothèse d'une culture spécifiquement anglo-saxonne et sud-africaine de la sécurité privée), il convient d'étudier les stratégies de légitimation auxquelles ont recours les entrepreneurs militaires mais aussi certaines bureaucraties gouvernementales ${ }^{8}$. Nous essaierons par conséquent de discuter de la pertinence des argumentaires utilisés par ces entreprises, ainsi que par leurs défenseurs qu'ils soient publics ou privés, pour justifier et légitimer leurs activités. Cela permettra de mettre en évidence les non-dits de ces discours de justification, mais aussi les éventuels décalages entre ces derniers et les pratiques sociales des entrepreneurs militaires. Nous partirons donc des discours de justification et de légitimation existants pour en montrer les erreurs de raisonnement, le manque de base éthique, ainsi que les omissions.

(Re-)définir l'identité sociale des entreprises de coercition para-privées : des entreprises « comme les autres »?

La légitimité accordée par certains aux entrepreneurs militaires ne découle pas de leurs actions de "sécurisation" dans un monde dans lequel les "nouvelles menaces" imposeraient aux gouvernements de déléguer leur monopole à des entrepreneurs privés. Au contraire, elle renvoie à des stratégies discursives qui tendent à vouloir imposer une certaine représentation de ces entreprises, ainsi que de leur utilité sociale. Or, comme l'a montré Murray Edelman, un discours politique ou sécuritaire doit toujours être analysé en relation aux " groupes cibles » auxquels il s'adresse et qui ont une sensibilité particulière à celui-ci ${ }^{9}$. Les « groupes cibles » du discours des entreprises 
de coercition comprennent notamment les agences de sécurité gouvernementales, mais aussi de plus en plus les bailleurs de fonds internationaux, d'autres entreprises ainsi que les actionnaires potentiels. Dans ce contexte, que ce soit sur les sites internet de ces entreprises ou dans les discours de leurs défenseurs, une première stratégie consiste à conforter la thèse selon laquelle il s'agirait d'entreprises " comme les autres ", et donc que la "privatisation de la sécurité » serait un phénomène banal. On insiste alors volontiers sur le fait que ces entreprises ont une identité managériale forte, qu'elles respectent tous les dispositifs légaux et financiers applicables aux entreprises, que certaines d'entre-elles sont cotées en bourse (Military Professional Ressources Incorporated - MPRI, ArmorsHolding) et qu'elles sont soumises et contraintes par la loi du marché.

Conformément à une certaine vulgate néo-libérale en vogue, la structure fortement concurrentielle de ce marché, accompagnée d'une certaine réglementation ainsi que de clauses contractuelles précises, assurerait alors le professionnalisme, l'efficacité (fondée sur le rapport qualité/prix) et le respect des législations en vigueur. Les «mauvais élèves » seraient automatiquement et immédiatement exclus et la gestion transparente favorisée ${ }^{10}$. Par exemple, ces entreprises ne travailleraient que pour des gouvernements reconnus par la communauté internationale (à l'exclusion de mouvements rebelles par exemple), seraient enregistrées légalement dans des Etats reconnus (fut-ce dans des paradis fiscaux), et se montreraient infiniment plus compétitives en termes de rapport qualité-prix que les opérations de l'ONU dont on aime à mettre en évidence les dysfonctionnements. Certaines d'entre-elles disposeraient même d'une doctrine militaire propre. L'internationaliste respectable et respecté Christopher Coker va même jusqu'à affirmer que le marché est éthique en soi puisque fondé sur des relations contractuelles contraignantes: "le commerce[de services militaires]a bien sûr été critiqué pour des raisons éthiques. Cependant, le marché est éthique : les entreprises tendent à remplir leurs obligations contractuelles à condition et dans la mesure où elles sont imputables vis-à-vis des clients $»^{11}$. La figure contemporaine de $l^{\prime}$ « entreprise responsable » joue donc un rôle structurant dans ce discours. Pourtant, l'idée que la logique du marché oblige ces entreprises à respecter les normes en vigueur et une certaine "image de marque ", et à laquelle on pourrait pourtant opposer de nombreux exemples ${ }^{12}$, repose sur un certain nombre de fausses évidences.

Il ne saurait être contesté que ces entreprises font preuve de professionnalisme, si l'on définit celui-ci en termes de capacités et de maîtrise des technologies de surveillance et de coercition militaire. Ainsi certaines, certes rares, sont capables de proposer des services spécialisés: capacités dans le domaine du «C3 I» (Command, Control, Communication, Information), transport lourd aéroporté, activités de formation à la guerre clandestine, opérations de contre-espionnage, opérations spéciales, services para-militaires, formation aux services médicaux et lutte contre la drogue (Executive Outcomes - EO dissoute en 1998, DynCorps, Sandline...). Certaines, dont EO, ont même été assimilées à des armées privées pouvant mobiliser des avions de combat, des blindés et des hélicoptères dans des opérations coordonnées. L'efficacité militaire à court terme de ces entreprises dans la mission qui leur a été confiée est souvent peu contestable. Mais justement, on ne saurait inférer la légitimité de l'efficacité militaire. L'évocation de l'efficacité permet surtout d'éviter celle de la base éthique de leurs actions particulières. Ainsi si toutes les entreprises se revendiquent de manière générale d'une éthique rigoureuse $\mathrm{e}^{13}$, du respect de normes internationales en vigueur 
et du code militaire, leurs pratiques sociales ne sont souvent pas à la hauteur de ces discours.

Le discours "managérial » omet de mentionner aux moins deux éléments conférant une certaine spécificité aux entreprises de coercition. Premièrement, du fait du lien entre sécurité et confidentialité, les clauses des contrats signés par ces entreprises ne sont que très peu souvent révélées. De la sorte, le principe de transparence peut être contourné sous prétexte de confidentialité d'informations «sensibles» relatives aux clients et à l'efficacité militaire de la mission. Ainsi on ne dispose que de très peu de détails sur les opérations menées par ces entreprises de coercition. Par exemple, on ne connaît pas avec certitude le rôle joué par MPRI dans l'offensive meurtrière de l'armée croate, qu'elle entraînait, au Krajina en $1995^{14}$. Le moins que l'on puisse dire est que cela ne pousse pas à accorder une confiance a priori à ces entreprises. Deuxièmement, plutôt que de groupes constitués et à l'identité définie, ces entreprises sont souvent sous-tendues par des réseaux de personnel (fondé sur l'appartenance passée à un même corps de l'armée, aux mêmes services gouvernementaux...) qui jouent sur des répertoires d'action et de légitimation différenciés, cela notamment au travers de la création de différentes vitrines légales. Si l'on peut y voir une logique de diversification économique, ces pratiques entrent aussi dans le cadre d'une logique d'évitement des conséquences néfastes de certaines actions à la moralité "douteuse ». Dans un tel contexte, distinguer les "bons élèves » des " mauvais élèves ", et a fortioriaffirmer que la logique du marché permettra d'éliminer les derniers au profit des premiers, est peu crédible. Un des avatars inquiétants de cette structuration en réseaux informels est certainement le lien entre entreprises de coercition et l'industrie de l'extraction de ressources naturelles.

S'il est peu aisé d'assimiler ces firmes à des " entreprises comme les autres ", c'est donc aussi parce que la nature du service proposé permet de concevoir des modes de rétribution peu orthodoxes. Ainsi, ces entreprises interviennent parfois en se faisant rétribuer par l'obtention de concessions minières ou pétrolières. Ces dernières sont revendues à des entreprises d'exploitation de ressources naturelles afin de rentabiliser l'opération. Ces pratiques sont facilitées par les liens financiers et personnels étroits existants entre certaines entreprises militaires et des entreprises d'extraction de ressources naturelles. Ainsi, dans certains cas, les entreprises de coercition ne se limitent pas au secteur de la sécurité, mais procèdent par des stratégies d'intégration verticale, stratégies qui reposent sur une synergie entre les secteurs de la sécurité d'une part et celui de l'extraction minière et pétrolière d'autre part ${ }^{15}$. L'enregistrement légal de ces différentes entreprises en tant que personnes morales distinctes ne fait alors que dissimuler l'existence de réseaux d'accumulation et de pouvoir plus vastes. Ainsi Sandline International et EO, qui ont entretenu des liens financiers et surtout personnels très étroits par le biais de l'homme d'affaire et ancien membre des Special Air Services(SAS) Anthony Buckingham, ont aussi été liées par une forte intégration financière et des personnels communs, à des entreprises d'extraction dont Branch Energy, Heritage Oil \& Gas et DiamondWorks. Ce n'est alors probablement pas un hasard que Branch Energy ait obtenu des concessions diamantifères importantes dans la région de Kono en Sierra Leone après que cette dernière ait été reprise au Revolutionary United Front(RUF) par EO en 1995. En 1992 ce fut Heritage Oil \& Gas qui fit venir l'entreprise militaire sud-africaine en Angola pour chasser l'UNITA du centre pétrolier de Soyo. De la même façon, l'intervention avortée de Sandline International sur l'île de Bougainville en Papouasie-Nouvelle-Guinée, qui fut en réalité sous-traitée au 
personnel de EO, avait vu Tim Spicer proposer au gouvernement local de payer l'entreprise en concessions minières ${ }^{16}$. Dans d'autres cas, on connait moins bien les réseaux personnels et financiers liant les entreprises de coercition à l'industrie minière et pétrolière. Mais les synergies entre ces deux secteurs d'activité dans des pays peu solvables demeurent à la base de certaines interventions. Ainsi, la société israélienne LevDan, dirigée par un ancien général qui a été impliqué indirectement dans les massacres de Sabra et Chatila, a obtenu en 1997 au Congo-Brazaville des parts d'un permis pétrolier avec deux entreprises pétrolières israéliennes, cela afin d'honorer le contrat de soutien au régime de Pascal Lissouba. Richard Banégas parle ainsi à juste titre de véritables "techniques modernes de prédation " qui se mettent en place au travers de ces stratégies de pillage des ressources du sous-sol impliquant à la fois des entreprises militaires, des entreprises d'extraction et le personnel des gouvernements locaux ${ }^{17}$. Il est dans ce contexte pour le moins étrange que ce fait soit utilisé par certains, dont David Shearer, pour souligner que l'intervention d'entreprises de coercition permet de renforcer les économies africaines en créant un climat de sécurité favorable aux investissements des industries de l'extraction minière et pétrolière ${ }^{18}$. Cet argument omet en effet, d'une part, que ce n'est pas un "climat " général mais des liens personnels et financiers spécifiques qui font intervenir ces entreprises d'extraction, et d'autre part que ces pratiques se nourrissent de la guerre en tant que mode d'accumulation primitive de capital en même temps qu'elles y participent.

Mais l'essentiel de cette représentation "managériale » des entrepreneurs militaires est probablement ailleurs que dans la négation des spécificités du «secteur de la sécurité ». En effet, elle s'inscrit dans une stratégie de communication qui consiste à se distinguer des «affreux» des années 1970, et du qualificatif de "mercenaire » qui l'accompagne. Ainsi si des gens comme Tim Spicer insistent sur le fait que leur entreprise dispose d'un vivier permanent de professionnels (sous-entendu comme toute entreprise), c'est pour se démarquer du mode de recrutement ad hoc attribué aux «mercenaires » du temps de la décolonisation ${ }^{19}$. Cela notamment pour ne pas tomber sous le coup de la législation internationale en vigueur ${ }^{20}$. Mais la distinction entre entreprises militaires privées et "mercenaires traditionnels" permet aussi de se débarrasser d'un certain nombre d'images négatives attachées à Bob Denard, Christian Tavernier ou Michael Hoare. L'enjeu de la labellisation joue donc un rôle crucial et fait porter le débat sur la question de savoir ce que sont ces «nouvelles" entreprises militaires, mais aussi sur ce qu'elles ne sont pas. La mobilisation de l'argument selon lequel il s'agirait d'entreprises " comme les autres" permet alors de montrer qu'elles se distinguent absolument des «affreux» qui, contrairement aux soldats privés modernes, n'auraient été recrutés que pour des missions ponctuelles, se seraient inscrits dans des réseaux plutôt que dans des structures stables et dont le comportement aurait été d'avantage mû par l'aventurisme et l'esprit de lucre que par le souci de légitimité internationale et l'ethos rigoureux propre aux militaires. Cette distinction est alors souvent assimilée à l'opposition pratique entre l'ancien (les « mercenaires ») et le nouveau (les entreprises militaires).

Si l'idée qu'il s'agisse d'entreprises responsables "comme les autres" relève certainement du mythe, celui-ci ne peut se maintenir que par la création d'un certain nombre de contre-mythes concernant aussi bien les pratiques sociales des mercenaires dits traditionnels que la prétendue nouveauté radicale du phénomène des entreprises militaires privées. Pourtant Bob Denard, dont personne ne conteste le caractère mercenaire, avait lui aussi déjà essayé de légitimer ses activités par des structures 
légales et commerciales lui permettant d'attirer des investissements étrangers ${ }^{21}$. Ainsi, il avait notamment créé une entreprise de sécurité (La société générale comorienne, SOGECOM) aux Comores, chargée de protéger des hôtels tout en lui permettant d'avoir à sa disposition un vivier permanent de professionnels pour d'autres missions éventuelles. De la même façon, il faut bien remettre en cause le discours sur la nouveauté radicale des entreprises militaires privées. Ces dernières s'inscrivent dans une histoire plus longue qui efface partiellement la distinction entre anciennes et nouvelles modalités de la sécurité privée (en tout cas si l'on considère la chute du mur de Berlin comme le moment charnière). Ainsi, les entreprises américaines Vinnel et Pacific Engineers \& Architects (PA\&E) étaient déjà intervenues aux côtés de l'armée américaine lors de la guerre du Vietnam. D'autres entreprises de sécurité militaire existaient également avant le tournant supposé des années 1990. L'entreprise britannique Security Advisory Services Ltd (SAS Ltd) fût créée en 1970. En 1967, le fondateur des Special Air Services (SAS), David Sterling, créa l'entreprise Watchguard International Inc. dont le but était de fournir des régimes, essentiellement dans le Moyen-Orient, en ex-militaires britanniques. Keenie Meenie Services (KMS), spécialisée dans la guerre contre-insurrectionnelle, a quant à elle été créée en 1974.

De la même façon, la distinction entre "mercenaires traditionnels " et «entreprises modernes " doit être nuancée par le fait qu'au-delà du caractère formellement institutionnalisé des entreprises de coercition para-privées, ces dernières s'inscrivent également dans des réseaux informels de professionnels de la sécurité. Ainsi, si les entrepreneurs militaires proclament souvent attacher une grande importance dans le recrutement à l'éthique des postulants, au respect du code militaire (cela en adoptant des critères de sélection des plus strictes : casier judiciaire vierge, tests psychologiques, carrière militaire honorable), le recrutement semble souvent se faire de manière informelle sur la base de la seule appartenance à un réseau. Réticularité et institutionnalisation vont ici de pair, ce qui doit considérablement relativiser la distinction entre mercenaires et entreprises militaires. Ainsi Richard Banégas peut souligner que "plutôt que de véritables armées privées, il s'agit(...)de réseaux informels de combattants et de techniciens rapidement mobilisables ${ }^{22}$.

La volonté de se démarquer du "mercenariat traditionnel » conduit également à des positionnements différenciés, voire antagonistes, entre ces entreprises. Cela se comprend aisément dans un contexte dans lequel la légitimité des ces entreprises est fragile et que certaines s'exposent plus que d'autres à l'accusation de mercenariat. Notamment celles s'étant engagées directement dans le combat comme EO ou Sandline International. Ainsi, on distingue souvent les entreprises militaires privées (PMC) des entreprises de sécurité privées (Private Security Companies,PSC), les premières fournissant des services militaires à des gouvernements, les deuxièmes assurant la sécurité d'autres entreprises ou organisations (Defence Systems Limited, DSL, filiale de la firme américaine ArmorsHolding). A l'intérieur de cette première catégorie on distingue alors fréquemment les " actives », qui s'engagent directement dans le combat et les "passives » qui se contentent de fournir logistique, entraînement, conseil et autres services non-combattants (MPRI, Vinnel, KBR). Ces distinctions structurent les identités de ces entreprises, ainsi que leur mode de légitimation. Ce dernier consiste en effet fréquemment à se démarquer des entreprises des autres catégories, supposées plus suspectes de mercenariat. Cela explique pourquoi les dirigeants de MPRI affirment ne jamais s'engager directement dans le combat (cela pour se démarquer d'entreprises 
comme Sandline International ou EO) et ceux de DSL ne jamais porter d'armes sur le terrain (cela pour se démarquer d'entreprises comme MPRI ou Vinnel).

Cependant, ici aussi il faut se garder d'adopter ces distinctions et ces qualifications en tant que telles. En effet, même si ces différentes catégories (dont la dénomination exacte peut changer selon les auteurs) reposent sur des créneaux commerciaux distincts, et qu'il faut se méfier de toute agrégation statistique qui ne tienne pas compte des spécificités, l'utilisation commune de technologies de coercition et de surveillance traditionnellement considérées comme militaires crée un continuum technologique qui produit des effets réels sur le terrain. Le recours à un même personnel, souvent issu des forces spéciales, génère des passerelles entre les différents métiers (gardiennage, gardes du corps, surveillance, logistique militaire). Il en résulte que les contraintes sur le terrain peuvent faire basculer les "entreprises de sécurité " d'une posture de dissuasion vers une posture de guerre offensive ${ }^{23}$. De la même façon, la stratégie d'intégration financière de différentes entreprises mobilisant un même personnel au sein de conglomérats multipositionnés ${ }^{24}$, fait que la présence $d^{\prime}$ '« entreprises de sécurité » peut favoriser celle d'entreprises plus offensives. Enfin, on peut se demander dans quelle mesure la distinction entre entreprises de conseil et de formation "passives" et entreprises "actives» est pertinente lorsque les « conseillers » sont présents sur le front et dirigent ainsi directement les opérations sur le terrain: selon certaines sources, des officiers de MRPI auraient été présents auprès des troupes croates lors de l'épuration ethnique du Krajina.

« Private Peace Enforcers » ou fauteurs de guerre?

Dans une configuration internationale dans laquelle la guerre ne fait plus partie (sauf exception) des options légitimes à la disposition des Etats, les entreprises de coercition para-privée ne peuvent démontrer leur utilité sociale qu'au travers de l'argument du maintien, du soutien et de l'imposition de la paix. Cela est d'autant plus le cas que les différentes institutions de l'ONU, ainsi que les ONG humanitaires, semblent constituer un des "groupes cibles" importants des pratiques discursives des entreprises de coercition. Elles représentent des clients potentiels, mais aussi et surtout leur recours éventuel à ces entreprises constitue une ressource symbolique importante. L'argument de la paix est ainsi un référent central dans les stratégies discursives de la majorité de ces entreprises. Cependant, il a été le plus développé par David Shearer, chercheur associé au International Institute for Strategic Studies (IISS), ancien employé du HCR et - semblerait-il - proche ami de Tim Spicer. Dans l'ensemble de ses articles, Shearer martèle son argument fondamental, mais simple, selon lequel toute résolution véritable d'un conflit ne peut passer que par le recours à la force, la seule réconciliation par la médiation étant jugée systématiquement insuffisante. Dans un contexte dans lequel les gouvernements et les organisations internationales seraient peu enclins à adopter cette première solution, trop coûteuse à tous les points de vue, il serait alors devenu souhaitable et même nécessaire de déléguer ce type de tâches à des entreprises privées étroitement régulées et contrôlées. Cela lui permet alors d'affirmer que " tout refus de coopérer avec ces entreprises fait encourir le risque de se priver de leur potentiel à aider dans la résolution de conflits apparemment insolubles $»^{25}$. Ainsi, ces entreprises seraient l'instrument idoine pour pallier les faiblesses du système onusien dans le domaine de l'imposition de la paix, mais aussi du maintien et de la consolidation de la paix, y compris dans sa dimension humanitaire.

L'analyse de Shearer se fait plus subtile quand il affirme que les effets potentiellement destructeurs de ces entreprises, qu'il reconnaît, doivent conduire à en réguler et 
encadrer étroitement les activités. Or, cela n'est possible qu'à condition de les reconnaître en tant qu'acteurs au moins potentiellement légitimes ${ }^{26}$. On est donc face à un dilemme moral: soit on refuse ces entreprises potentiellement dangereuses, les poussant ainsi à la clandestinité et donc à la liberté d'action la plus totale, soit on les régule pour en limiter les effets, auquel cas on doit au préalable les créditer d'une certaine légitimité. On retrouve ici en filigrane la distinction weberienne entre une "éthique de la conviction", amenant à agir selon les sentiments sans référence aux conséquences, et une "éthique de la responsabilité », interprétant l'action en termes de moyen-fin et devant être à la base de l'action politique. En posant le problème ainsi, il va de soi que Shearer privilégie la posture de l'« engagement constructif » avec ces entreprises. Shearer reconnaît également volontiers que la logique de ces entreprises étant celle du seul profit, elles sont amenées à privilégier la stabilisation à court terme au détriment de la résolution des problèmes structurels sous-jacents aux conflits: l'étroite coopération entre entreprises militaires privées et institutions chargées de la « reconstruction de la paix » dans une optique de long terme n'en est que d'autant plus nécessaire.

Toutes les grandes entreprises se sont engouffrées dans la brèche ouverte par Shearer en affichant leur capacité à agir en faveur de la paix, des opérations humanitaires et des institutions multilatérales (et notamment des institutions de Bretton Woods) ${ }^{27}$. EO avait d'ailleurs déjà perçu les atouts du "marché de la paix ", bien avant les écrits de Shearer, son PDG Eeben Barlow n'hésitant pas à se présenter comme un " private peaceenforcer » dès le début des années 1990. Depuis, l'entreprise britannique DSL, réputée favorite du système onusien, aurait travaillé pour l'organisation elle-même, mais aussi pour sept de ses agences et un certain nombre d'ONG. De la même façon, des entreprises comme PA\&E et International Charter Inc. (ICI) auraient travaillé pour le compte de l'ECOMOG en Sierra Leone et au Liberia, quant à Sandline International, elle se vante d'avoir travaillé avec des ONG comme MSF, CARE, Caritas et World Vision. Selon un auteur: "A un certain nombre d'occasions, les nations unies et les organisations régionales de maintien de la paix ont utilisé silencieusement des entreprises militaires privées dans le combat direct ${ }^{28}$. Il est difficile de vérifier la véracité de ces affirmations, mais il importe ici de souligner qu'elles s'inscrivent notamment dans une stratégie consistant à essayer de susciter un transfert de légitimité des institutions multilatérales et des ONG humanitaires vers l'industrie militaire privée. Preuve que la paix est devenue un argument porteur, un groupe d'intérêt (The International Peace OperationsAssociation, IPOA) s'est même constitué aux Etats-Unis, rassemblant le noyau dur de la sécurité militaire privée américaine (PA\&E, ICI, MPRI, Airscan International, TASK International...) afin de promouvoir des normes industrielles " au service de la paix ». L'IPOA a ainsi développé un code de conduite affirmant les principes de transparence et de responsabilité, ainsi qu'un plan de soutien à la MONUC en RDC. Son président, Doug Brooks, a établi un parallèle entre son organisation et les ONG humanitaires en affirmant que "gagner sa vie et en même temps sauver des vies n'est pas une chose mauvaise indépendamment de savoir si on travaille pour une ONG ou une entreprise militaire privée " ${ }^{29}$. Outre le fait que ces contrats au service d'opérations de paix semblent être largement surexposés par rapport à l'ensemble des pratiques sociales de ces entreprises, l'argumentation de Shearer repose sur des bases théoriques très fragiles (voir infra). Cependant, conformément aux objectifs de cette partie, nous nous contenterons ici de montrer les décalages entre les discours et certaines pratiques sociales sur le terrain. En effet, ces décalages semblent indiquer que ces entreprises peuvent tout autant être 
des vecteurs belligènes que des «peace-enforcers». Ainsi, leur objectif serait de contribuer à la stabilisation de situations politiques fragiles et en aucun cas de contribuer à la déstabilisation de régimes en place. Pourtant les contre-exemples abondent. Ainsi, en 1995 l'entreprise de déminage américaine Ronco aurait fourni en armes des militaires rwandais en violation à l'embargo international sur le Rwanda et le Burundi ${ }^{30}$. Cette opération de soutien à Kabila et Kagamé aurait été menée par le biais d'anciens militaires américains des Special Operation Forces travaillant pour l'entreprise dans l'objectif (atteint) de renverser Mobutu. L'entreprise AirScan quant à elle aurait, selon Kevin O'Brien, été impliquée dans des transferts d'armements de l'Uganda au Soudan en soutien à la rébellion du SPLA. Enfin, Sandline International a de manière très officielle proposé de soutenir l'UCK au Kosovo en 1997. Indépendamment de savoir si les objectifs visés par ces actions sont intrinsèquement légitimes ou pas, il convient de noter qu'elles s'apparentent davantage à une ingérence militaire dans les affaires d'un pays tiers, et donc à un acte d'agression selon le droit international (s'il s'avérait qu'un Etat se trouve derrière ces interventions, ce que les sources respectives semblent suggérer), qu'à à une quelconque « opération de paix ».

Les méthodes utilisées seraient également souvent peu compatibles avec les discours policés de ces entreprises. Ainsi, selon Philippe Chapleau et François Misser (citant un rapport publié par trois ONG canadiennes), la compagnie canadienne John Van Nostrand Associates Ltd aurait été engagée par une entreprise sud-africaine au Ghana pour relocaliser de force 20000 personnes, ce qui aurait donné lieu à la destruction de maisons et d'écoles et l'interdiction de cultiver la terre. Dans les affrontements violents qui s'en sont suivis plusieurs civils auraient été tués ${ }^{31}$. De sources diverses, l'opération de EO en soutien au gouvernement du MPLA en Angola en 1994 aurait impliqué l'utilisation de bombes incendiaires (de type napalm) contre les zones contrôlées par l'UNITA. Même si EO a par la suite été accréditée d'avoir par ce biais favorisé les négociations en vue des accords de Lusaka, on peut se demander dans quelle mesure l'utilisation de telles bombes est compatible avec la reprise d'un discours onusien. La même chose peut être dite des milliers de mines anti-personnelles que EO auraient disséminées en 1995 autour de la concession diamantifère de Kono en Sierra Leone. Enfin, rappelons le rôle probable joué par MPRI dans l'offensive operation stormmenée par l'armée croate en Krajina en août 1995. Celle-ci donna lieu à de véritables épisodes de nettoyage ethnique qui firent plus de 170000 réfugiés, plusieurs centaines de morts et des recommandations d'incrimination de généraux croates pour crime de guerre auprès du TPIY ${ }^{32}$. MPRI semblerait avoir été étroitement impliqué dans cette opération. En effet, des dirigeants de l'entreprise auraient eu une dizaine de rencontres avec des officiers croates impliqués à Krajina pendant les 5 jours avant l'offensive et les armes des forces croates auraient été fournies par un marchand d'arme proche de la direction de MPRI ${ }^{33}$. Selon certains observateurs, des employés de MPRI auraient même participé directement à l'offensive ${ }^{34}$. Si le commerce d'armes est généralement présenté comme ne faisant pas partie de services proposés, la réalité est autre. Ainsi, en 1998 fut révélé que Sandline International avait vendu des armes aux forces du gouvernement déchu de Kabbah en Sierra Leone en 1997 en violation à l'embargo de l'ONU sur le pays. Finalement, l'entreprise fut disculpée dans ce Arms-to-Africa Affair d'avoir agi sans l'aval du Foreign and Commonwealth Office (FCO). Il n'en reste pas moins que sa capacité réelle à fournir en armes des forces armées sur un territoire sous embargo international fut ainsi révélée. Ainsi, loin de se contenter de renverser le rapport de force sur le terrain, ces entreprises peuvent durablement accroître le niveau de violence en surajoutant à 
des dynamiques conflictuelles locales des moyens technologiques relativement sophistiqués. Cela est d'autant plus vrai que fonctionnant sur la base de la logique du profit, ces entreprises sont peu incitées à imposer une limite à leurs ventes d'armement. Les liens entre entreprises de coercition para-privées et marchands d'armes sont nombreuses: Gurka Security Guards a travaillé en étroite collaboration avec J\&S Franklin, DSL travaille avec Heckler et Koch, MPRI avec le fournisseur d'armes Cypress International et Vinnel est une filiale de BDM International (dont la vente d'armes est une activité importante). Le "transfert de technologie ", euphémisme s'il en est, qui en résulte, augmente les capacités de violence des acteurs en présence et peut ainsi nourrir le conflit. Ainsi, à la fin des années 1980, la firme LIAT Finance and Construction serait intervenue en Sierra Leone en se faisant payer au travers de l'octroi de concessions diamantifères. Celles-ci auraient par la suite été utilisées pour le commerce illégal de diamants servant à acheter des armes venant nourrir le conflit ${ }^{35}$. Ces dynamiques polémogènes ne doivent pas étonner dès lors qu'il s'agit d'organisations lucratives ayant un certain intérêt à la prolongation et à l'intensification des conflits. Elles obligent, dans tous les cas, à relativiser le discours sur les entrepreneurs militaires comme vecteurs de «stabilité » et de " paix ».

Contrôler les entrepreneurs militaires pour mieux les contenir?

$\mathrm{Au}$ sein de la communauté académique et politique un consensus semble aujourd'hui se dégager sur l'idée que, étant donné qu'une interdiction pure et simple ne saurait être effective, il importe aux gouvernements et aux organisations internationales de mettre en place des modes de régulation du phénomène afin de l'encadrer tout en le légitimant. Cela permettrait, pense-t-on, de réduire les effets destructeurs de la mise à disposition de technologies de coercition et de surveillance de type militaire à des acteurs privés. L'argument implicite est que si les entreprises de coercition peuvent avoir des effets néfastes là où elles interviennent, ce n'est que parce qu'elles sont insuffisamment contrôlées par les gouvernements occidentaux. De ce fait des analystes comme Kevin $O$ 'Brien, qui dans certains de ses écrits s'est montré très critique à l'égard des entreprises de coercition, évoluent vers une position de plus en plus complaisante à l'égard du phénomène sous prétexte que les gouvernements peuvent le contrôler ${ }^{36}$. Or, cet argument omet que certaines bureaucraties gouvernementales ont un intérêt certain aux agissements des entreprises de coercition avec lesquelles elles entretiennent des liens étroits au travers de réseaux de professionnels qui chevauchent la frontière public/privé. L'existence de telles entreprises permet à certains professionnels "publics» de la sécurité de poursuivre leurs activités, tout en maintenant la possibilité de nier leur implication (argument duplausible deniability). En d'autres termes, l'émergence des entreprises de coercition ne s'est jamais faite en rupture avec les services gouvernementaux. Ces entreprises ne sont pas le contrepoids privé de la coercition étatique, mais une des ressources offertes à certains réseaux de professionnels de la sécurité qui savent jouer sur les deux registres d'action, privé et public. Dès lors, il est peu probable que la seule régulation des entreprises de coercition par les gouvernements, ou sous le contrôle de ceux-ci, puisse permettre de juguler les effets néfastes du phénomène.

Susan Strange a déjà montré combien la distinction entre politique et économie est simpliste si on la réduit à la distinction entre Etat et marché. En effet, les structures politiques (L'Etat) sont traversées par des processus économiques de la même façon que les structures économiques (les entreprises) sont traversées par des processus politiques $^{37}$. Mais il nous faut ici aller plus loin pour montrer comment la distinction 
même entre Etat et marché, que Strange reproduit, est mise en échec par l'existence de champs de professionnels qui cassent cette distinction, notamment dans le domaine de la sécurité. Comme l'a analysé Béatrice Hibou, la privatisation de fonctions étatiques, bien loin de se réduire à « un retrait de l'Etat », perpétue le pouvoir des élites politiques en leur permettant de renégocier les frontières officielles du public et du privé à leur avantage. La privatisation est ainsi un mode particulier de "gouvernementalité » au sens foucaldien du terme. En effet, elle est l'occasion d'un «redéploiement de l'Etat » par le truchement d'acteurs dits privés: notamment par le biais de stratégies de délégation, d'extension des réseaux de patronage et de chevauchements par les élites gouvernementales de la frontière public/privé. Elle utilise le concept paradoxal de "privatisation de l'Etat» pour décrire le brouillage des frontières entre l'Etat et le marché qui en résulte ${ }^{38}$. A notre sens cette dénomination pose problème dès lors qu'elle repose sur une vision essentialisée de l'Etat (l'Etat reste Etat malgré toutes les reconfigurations des rapports de pouvoir) et qu'elle confond gouvernementalité, gouvernement et Etat. Cependant elle ouvre des perspectives d'analyse en interdisant de faire une distinction claire entre le secteur public et le secteur privé, tout en permettant d'analyser leur interface.

La première implication de cette grille de lecture, qui s'applique bien à la "privatisation» de la sécurité, est que les entreprises militaires existantes, qu'elles entretiennent ouvertement des liens étroits avec leur Etat d'origine (notamment les grandes entreprises américaines), ou qu'elles affichent une relative autonomie (cas britannique, sud-africain...), sont toutes sous-tendues par des réseaux de professionnels étroitement liés aux bureaucraties et aux services gouvernementaux. Il n'est pas possible ici de faire une sociologie détaillée de ces réseaux. Contentons-nous ici de souligner qu'ils impliquent à un premier niveau les professionnels "publics » de la sécurité, souvent au travers des unités spécialisées dans les opérations commando (Special Operation Forces,SAS britanniques, légion étrangère française), mais aussi au travers des grandes agences de sécurité et notamment les services secrets.

Ainsi, dans un contexte de réduction des effectifs, elles permettent notamment à certains militaires de se recycler dans le privé, tout en continuant à œuvrer dans la même profession. Le secteur privé devient alors un garant de sécurité de l'emploi, tout en permettant dans certains cas de retirer des bénéfices autrement plus importants que dans le secteur public. Qui plus est, comme l'a montré le cas du trafic de femmes dans lequel fut impliqué DynCorps en Bosnie-Herzégovine, les crimes commis par les employés des entreprises militaires privées ne relèvent pas de procédures judiciaires prévues dans le cadre de l'appareil militaire, mais du droit local. Les employés de DynCorps concernés par cette affaire s'en tirèrent ainsi avec un simple licenciement. Le statut privé est donc moins contraignant que celui de militaire. Enfin, comme l'ont montré les opérations en Afghanistan et en Irak, la privatisation de la sécurité a l'avantage de permettre d'externaliser certaines fonctions considérées comme n'étant pas au cœur du métier militaire ("contrôle des foules", soutien humanitaire, gardiennage), cela afin de libérer de la main d'œuvre militaire pour les opérations de combat. Ce n'est alors pas un hasard si les QG de ces entreprises sont souvent localisés à côté de grandes bases militaires.

A un deuxième niveau, elles impliquent souvent des professionnels de la politique au sein des ministères des Affaires Etrangères et de la Défense. Dans le cas américain, les entreprises de coercition sont ainsi soumises à un système de licence, contrôlé par le Département d'Etat et le Pentagone, sur le même mode que la vente d'armements. Dans 
le cas de la Grande Bretagne, le FCO disposerait d'une liste de toutes les entreprises pouvant être mis à sa disposition pour accomplir des missions ne pouvant, pour des raisons de légitimité politique, être directement assumées par les pouvoirs publics. Ainsi l'entreprise KMS aurait en 1993 entrainé l'armée Sri Lankaise à la guerre contreinsurrectionnelle pour la soutenir dans la guerre contre les Tigres Tamouls. Cette opération aurait été jugée trop sensible pour être assumée directement par le gouvernement britannique. De la même façon, Saladin Security aurait entraîné les troupes du Sultanat d'Oman. Ces pratiques ont aussi comme avantage de permettre de contourner le contrôle parlementaire et ainsi de court-circuiter les mécanismes de prise de décision habituels : dans le cas américain, le congrès n'a un droit de regard que sur les contrats d'un montant supérieur à 50 millions de dollars. Cela laisse donc une marge de manœuvre relativement importante à certains réseaux de professionnels au sein du Secrétariat d'Etat et du Pentagone.

Cette "privatisation de la politique étrangère " permet aussi de contourner l'opinion publique, fait bien perçu par ces entreprises qui en font un argument de leur stratégie de marketing. Enfin, ces entreprises permettent à certaines bureaucraties gouvernementales de contourner les règles internationales en vigueur. Ainsi, le contrat d'entraînement de l'armée de la Fédération Croato-Musulmane par MPRI en 1996 permit au gouvernement américain de s'impliquer dans les opérations de maintien de la paix dans le cadre de l'IFOR en Bosnie, qui en toute rigueur l'obligea à une certaine neutralité, tout en soutenant l'armée de la fédération. Ces entreprises s'inscrivent donc bien dans une forme de gouvernementalité qui permet de contourner les contraintes démocratiques et juridiques, et cela malgré l'existence de régulations relativement rigides. En effet, les Etats-Unis sont souvent cités comme exemple de régulation stricte de l'intervention des entreprises de coercition para-privées. On pourra alors dire que les entreprises de coercition, "libres de souveraineté » selon l'expression de James Rosenau, permettent aux gouvernements d'échapper aux contraintes de leur propre souveraineté39.

Il ne s'agit ici en aucun cas de reproduire les thèses d'une certaine sociologie marxiste sur le «complexe militaro-industriel» ou l'« élite du pouvoir» (Wright-Mills) selon lesquelles il y aurait une convergence étroite d'intérêts de toutes les fractions associées à l'exercice du pouvoir ${ }^{40}$. Au contraire, ces différents réseaux transversaux doivent être analysés de manière différenciée et dans leurs logiques propres. La sécurité privée de type militaire se structure en réseaux différenciés qui sont en compétition. Il n'y a pas d'alliances objectives. Il est certes très difficile de cartographier avec certitude les différents réseaux existants mais soulignons, pour dissiper toute accusation de monisme sociologique, qu'il a existé des tensions importantes entre Gurkha Security Group et Sandline International. Ainsi, lorsque l'opération de cette dernière sur l'île de Bougainville en Papouasie-Nouvelle-Guinée se solda par un échec cuisant du fait de l'opposition de l'armée locale, l'enquête qui s'ensuivit révéla que Gurkha Security Group avait financé l'opposition par le biais de l'entreprise d'armement J\&S Franklin. De la même façon, le arms-to-africa-affairdans lequel Sandline International fut impliqué, révéla que le High Commissionerbritannique (l'équivalent de l'ambassadeur dans les pays du Commonwealth) P. Penfold, ainsi que les services secrets britanniques, avaient travaillé étroitement avec l'entreprise. Cependant toute une partie du FCO, dont le ministre des Affaires Etrangères lui-même, avait été tenue à l'écart de cette coopération. 
Si les réseaux de professionnels de la sécurité et de la politique qui sous-tendent les entreprises de coercition impliquent donc souvent les bureaucraties gouvernementales, ces entreprises ne peuvent être considérées comme de simples instruments d'un gouvernement unitaire. Cela est d'autant plus le cas, que rien ne permet d'affirmer que les professionnels de la politique sont en position dominante à l'intérieur de ses réseaux : les forts liens entre entreprises de coercition et entreprises d'extraction de ressources naturelles semblent même suggérer le contraire. Il faut donc souligner que réfléchir en termes de réseaux transversaux à la frontière privé/public, n'est pas s'inscrire dans une lecture stato-centrée des relations internationales, mais au contraire déconstruire l'Etat en réseaux de pouvoir s'inscrivant dans des champs de pratiques diverses, dépassant de loin le seul cadre institutionnel étatique et dans lesquels la position institutionnelle n'est qu'une ressource parmi d'autres.

Il découle de ce qui vient d'être dit que les effets destructeurs des entreprises de coercition dans les endroits où ils interviennent ne sont pas forcément liés à un manque d'implication des " pouvoirs publics ». Du point de vue pratique, cela implique que la seule régulation étatique de ces entreprises, qui instaurerait un droit de regard des services gouvernementaux, n'est pas forcément une panacée. En effet, comme le montre le cas américain, les termes dans lesquels des contrats spécifiques sont approuvés par les services gouvernementaux sont gardés secrets. La problématique n'est donc pas tant celle du contrôle des entreprises de coercition que celle du contrôle des gouvernements susceptibles de recourir à celles-ci pour intervenir dans un espace opaque échappant au contrôle démocratique. Ainsi, du fait des intérêts des professionnels de la politique à travailler de concert avec les entreprises de coercition, il est peu probable que ceux-ci acceptent de se soumettre à des modes de régulation absolument transparentes. Ceux-ci les priveraient de l'argument du plausible deniability en cas de "bavure » ou de "dérapage ». Cela vaut d'ailleurs aussi pour les hommes politiques des Etats dans lesquels les entreprises interviennent dans le cadre d'opérations extérieures. En effet, ces entreprises s'inscrivent aussi dans des réseaux de pouvoir locaux. Ainsi, le fils du président Arap Moi de Kenya ainsi que le demi-frère du Président Museveni auraient eu des liens avec EO en tant que représentants locaux de ses filiales (Saracen). Le caractère transnational de ces réseaux d'accumulation et de pouvoir limite ainsi aussi la portée des régulations nationales: en délocalisant leur siège social, ces entreprises peuvent jouer sur les différentiels de régulation. On comprend alors mieux pourquoi les entreprises elles-mêmes sont généralement favorables à une "régulation flexible» de leurs activités par les gouvernements. Elle leur permet de légitimer leur existence, tout en maintenant intacts leurs liens étroits mais relativement dissimulés, avec les bureaucraties gouvernementales.

Cette partie nous a permis de confronter les discours de justification des entreprises militaires de sécurité internationales à leurs pratiques sociales. Cela a montré qu'il n'est pas besoin de passer par des critiques naïves (risque que les entreprises militaires se retournent contre leurs Etats, soutiennent des actions terroristes, se livrent au trafic de drogue ou conduisent à un chaos généralisé du fait de l'implosion des Etats) pour remettre en cause leurs pratiques. Cependant, ce faisant nous sommes restés prisonniers de la logique interne de la stratégie de légitimation des entrepreneurs militaires. De plus, nous nous sommes bornés à étudier la portée des entreprises de coercition para-privées dans leurs formes et leurs répertoires d'action actuels, et qui pour certains d'entre eux peuvent sembler fortuits. De la critique des arguments de légitimation des entreprises de coercition, il nous faut donc passer à une critique 
beaucoup plus fondamentale de l'idée d'un marché de la sécurité (lorsque celle-ci est utilisée autrement que de manière métaphorique). Il nous faut donc aborder, dans une optique plus générale et plus théorique, les effets structurels que la prolifération de ce type d'entreprises risque d'avoir sur notre façon de penser le politique ainsi que de son rapport à la sécurité.

Vers une remise en cause fondamentale des entreprises de coercition para-privées : les effets structurels de l'institutionnalisation d'un marché de la sécurité internationale Jusqu'ici nous avons cherché à saisir la dynamique sous-jacente aux entreprises de coercition para-privées en évitant le double écueil de l'assimilation de celles-ci à l'Etat ou au contraire à la fin absolue du contrôle gouvernemental sur les moyens de la coercition légitime. Cependant nous ne saurons en rester à l'idée d'un "entre-deux " fondée sur l'interpénétration de la sphère publique et de la sphère privée. En effet, il y a bien une différence de nature et non de degré dans l'idée de sécurité privée par rapport à l'option théorique d'un monopole d'Etat. Cette différence repose sur la fausse évidence selon laquelle la sécurité serait un bien pouvant être échangé sur un marché suivant les fluctuations de l'offre et de le demande. De la même façon que Karl Polanyi avait montré les effets destructeurs de la constitution du travail, de la monnaie et de la terre en biens économiques suivant l'idéologie du marchéti ${ }^{41}$ il nous faut désormais montrer que la sécurité ne saurait être constituée en marchandise, au travers de l'institutionnalisation d'un marché de la sécurité privée, sans effets structurels majeurs et - pourrait-il sembler - peu souhaitables. Cela d'une part du fait du statut de la sécurité en tant que "speech-act", d'autre part du fait de la dimension politique du concept de sécurité et enfin parce qu'elle renforce la tendance à la dé-différenciation des champs de la sécurité intérieure et de la sécurité extérieure et lève ainsi un certain nombre de contrôles traditionnels sur l'utilisation de la violence.

La " sécurité privée » à l'épreuve des analyses de la sécurité en tant que pratique discursive

La sécurité ne saurait être considérée comme une "marchandise» ou un "service marchand " parce qu'elle ne se réfère pas à une situation objective qui existerait en tant que situation de sécurité préalablement à la labellisation de celle-ci en tant que situation de sécurité. Comme l'a montré Ole Waever, s'inspirant de la lecture de la pragmatique linguistique d'Austin par Derrida, la sécurité ne se réfère pas à un environnement de menaces objectives à la sécurité existant indépendamment de l'énonciation sécuritaire ${ }^{42}$. Au contraire, elle est construite par un acte de discours, un speech-act, qui ne se réfère pas à un objet de sécurité préexistant mais qui, au contraire, construit certains phénomènes (considérés par exemple comme simples risques) en tant que menaces à la sécurité. Il en découle qu'il faut dé-essentialiser la sécurité, c'està-dire ne pas la considérer comme une « chose » ou comme un «état de fait » existant indépendamment de l'énonciation. Evidemment, cela ne veut pas dire que les phénomènes sociaux qui sont sécurisés (groupes clandestins faisant usage de violence, meurtres, immigration...) n'existent pas avant l'énonciation sécuritaire, mais tout simplement que dans l'analyse de ces phénomènes en tant que problème de sécurité, c'est le langage qui constitue la réalité première. La sécurité, ainsi que la représentation des menaces à celles-ci, sont le produit de pratiques discursives qui confèrent une saillance politique particulière à certains phénomènes sociaux et légitiment par-là le recours à des pratiques d'exception qui excluent tout compromis politique. Plutôt que de parler de la sécurité, comme s'il s'agissait d'une réalité figée et objective, on devrait alors parler de processus de sécurisation et d'insécurisation ${ }^{43}$. Or, la commercialisation du label sécuritaire en tant que "produit» ou "service» 
constitue au contraire l'objectivation, la fétichisation la plus ultime du concept de sécurité.

Ce décalage risque alors d'avoir des effets structurels des plus concrets. En effet, la construction de la sécurité en tant que bien consommable et échangeable permet à l'offre de protection, à condition que celle-ci produise simultanément un savoir sécuritaire, de déterminer la demande de protection. Comme l'a souligné Anna Leander, la protection est un « service » qui, au travers du processus de sécurisation qui le sous-tend, peut créer sa propre demande ${ }^{44}$. Cela non pas au sens de la trop fameuse loi de Say, mais au sens où toute énonciation sécuritaire est en même temps révélatrice d'une certaine construction de la menace qui, dès lors qu'elle se traduit en savoir autorisé, produit effectivement un sentiment d'insécurité qui demande à être comblé. Il devient maintenant évident que la sécurité ne saurait être l'objet d'un marché sur lequel se rencontrerait une offre et une demande autonomes. En effet, par la force de l'acte de langage qui sécurise un phénomène, tout en insécurisant ainsi l'opinion publique, l'offre et la demande de sécurité sont littéralement insécables. Elles relèvent toutes deux d'un même processus d'(in)sécurisation. Ainsi, si l'acteur qui offre la protection est aussi capable de contrôler le processus d'(in)sécurisation, il peut également déterminer la demande de protection.

Pour compléter cette analyse deux éléments doivent être précisés. Contrairement à ce que suppose l'approche deridéenne de Weaver, la simple structure performative du langage ne suffit pas à sécuriser et insécuriser. Il faut que le discours soit effectivement accepté comme légitime et comme l'expression d'un savoir autorisé. Dès lors, comme l'a montré Bourdieu, le discours, y compris dans son aspect performatif, doit être analysé de manière contextualisée, et notamment en tenant compte de la position au sein de l'espace social de ceux qui énoncent ${ }^{45}$. Or, de ce point de vue, le personnel des entreprises de coercition qui est chargé de la formation, du conseil et de l'entraînement de services de sécurité étrangers, est idéalement positionné pour produire un savoir autorisé puisque ses membres sont engagés en tant que spécialistes reconnus de la sécurité. Ces derniers peuvent ainsi déployer des discours de sécurisation qui produisent la demande à laquelle elles sont censées répondre. Il est ainsi facile pour Vinnel et Strategic Applications International (SAIC) chargées de la formation des forces de sécurité saoudiennes, ou DynCorps entraînant les forces de la «nouvelle " police irakienne, de promouvoir des fantasmes sécuritaires liant notamment les prétendus réseaux de «terrorisme international » et des groupes de résistants locaux, cela afin de justifier leurs activités de formation et en susciter la demande ex post. En déterminant contre quelles menaces il convient effectivement de savoir se défendre, elles déterminent aussi quelle offre de protection la demande doit rechercher.

On pourrait ici rétorquer que la position sociale de ce personnel ne diffère pas des conseillers militaires envoyés directement par les ministères de la Défense. Cependant, deuxième élément, dans la mesure où il s'agit d'entreprises privées, agissant notamment dans une logique de profit, elles sont davantage tentées que des conseillers publics d'étendre l'« environnement des menaces » à la sécurité. En effet, alors que ces derniers vont vraisemblablement promouvoir leurs lectures sécuritaires du monde afin de conforter leur utilité sociale, les entreprises de coercition ont un intérêt existentiel et financier à vendre les peurs à laquelle "répond» leur capacité à rassurer et à protéger. Le caractère autoréférentiel de la notion sécurité, ainsi que son affinité avec l'urgence politique, place ainsi structurellement les entreprises dans une position de force sur le «marché de la sécurité » en leur permettant, dans une certaine mesure, 
d'orienter la demande (en permettant de distinguer les vraies menaces des fausses menaces) et d'en déterminer le "volume" (par un processus d'extension de l'environnement des menaces à la sécurité). Cela ouvre la possibilité à de nombreux rackets. En effet, comme l'a souligné Charles Tilly: "L'idée que l'on aura du motprotectiondépend principalement de l'estimation que l'on fait de la réalité et de l'extériorité de la menace. Celui qui produit à la fois le danger et la défense, payante, contre celui-ci est un racketteur $\aleph^{46}$.

La sécurité ne saurait être assimilée à un bien "comme les autres " pouvant être échangé sur un marché parce que l'offre et la demande y relèvent d'un même processus de sécurisation et d'insécurisation et non de logiques autonomes. L'offre de protection peut y susciter la demande plus que dans d'autres types de secteurs. Cela non seulement par le biais du "dilemme de sécurité » (selon lequel accroître les capacités défensives d'un acteur insécurise souvent d'autres acteurs qui ont ainsi en retour un besoin de protection), mais surtout par le biais de l'acte de langage que constitue l'énonciation sécuritaire. Certains diront pourtant que ces entreprises ne sont pas toujours en position de produire un savoir sécuritaire. En effet, dans certains cas, ils ne sont engagés que pour accomplir des missions préconçues par d'autres acteurs, notamment en vue d'un retour à la paix. C'est à cet argument qu'il faut désormais s'intéresser.

La dimension politique du concept de sécurité et les travers de l'idée de privatisation des opérations de paix

Les entrepreneurs de coercition ne représentent pas la solution idoine aux « nouveaux conflits internationaux ». Il importe ainsi de montrer en quoi l'analyse de Shearer est biaisée. En effet son approche volontairement favorable aux entreprises militaires part d'un postulat de base qui est qu'il faut « aborder les entreprises militaires comme un système $d^{\prime}$ armes $\aleph^{47}$. Cette perception purement instrumentale et technique des entreprises militaires suppose que le recours à la coercition produise les mêmes effets indépendamment de l'identité des acteurs qui y ont recours. Elle part de l'idée que le pouvoir, en l'occurrence de coercition militaire, est une capacité quantifiable et qu'il convient simplement, face à une situation conflictuelle donnée, d'identifier l'acteur qui en a une capacité suffisante. Or, comme l'a montré Michel Foucault, le pouvoir est un processus relationnel et non une pure capacité ${ }^{48}$. On peut en inférer qu'il ne produit pas les mêmes effets selon les acteurs impliqués puisqu'une relation sociale ne sera pas la même selon l'identité des acteurs qui entrent dans la relation. Une conception "technique" (par opposition à une conception "politique ») du recours aux entreprises de coercition serait alors celle qui postule que l'on peut distinguer le recours à la violence de la perception qu'ont les acteurs concernés de ce recours et de ceux qui y ont recours. Or, il n'en est rien. La résolution de conflit n'est pas un problème technique dans lequel on ajuste des capacités militaires de façon à produire une situation de paix, mais un processus politique dans lequel des processus de légitimation et de délégitimation entrent également en jeu. Ainsi comme l'a montré John Crowley, toute sociologie positiviste excluant la dimension de l'éthique et de la légitimité de la régulation des conflits, est aussi incapable de concevoir un retour à la paix. Il faut donc mettre un accent particulier sur l'identité sociale des acteurs qui prétendent mettre fin aux conflits ${ }^{49}$.

Avoir recours aux entreprises de coercition pour résoudre des conflits, c'est postuler que la sécurité relève d'un problème technique et que, de ce fait, elle peut être assumée 
par le marché. C'est précisément parce que les violences protéiformes dans les pays du sud sont trop souvent considérées comme un problème technique, et en tout cas apolitique, que l'idée du recours à des entreprises de coercition a pu avoir un tel succès. En effet, ces entreprises se présentent souvent comme spécialisées dans les « conflits de basse intensité » caractérisés par une violence dérégulée, irrationnelle, insaisissable et chaotique. Or ces conflits s'inscrivent au contraire dans des processus de légitimation et de délégitimation politique dont il convient de comprendre les ressorts. La seule compétence technique dans un certain type de conflit ne saurait y mettre fin. En ayant recours aux entreprises militaires, on esquive le problème de la légitimité au profit de celui de la seule efficacité. Or, cette erreur, qui est celle de Shearer, induit des effets non souhaités. D'une part parce qu'en adoptant une posture de techniciens dans la résolution de conflit, ces entreprises ne sont pas susceptibles de tenir compte des effets politiques pourtant réels de leurs actions, d'autre part parce qu'en tant qu'organisations lucratives exogènes aux conflits politiques elles ne sont pas considérées comme légitimes par les acteurs impliqués à les résoudre, serait-ce par la force. L'idée de "marché de la sécurité » omet d'analyser le concept de sécurité dans son rapport au politique pour ne voir que sa seule dimension technique, militaire et stratégique. Comme l'ont montré des auteurs comme Ron Lipschutz, le concept de sécurité est pourtant constitutif du politique en ce sens que toute lecture sécuritaire contient aussi de manière sous-jacente une certaine représentation de la communauté politique qu'il convient de défendre ${ }^{50}$. De plus, il « définit la place où apparaissent dans l'espace public les acteurs ainsi que les institutions politiques et sécuritaires qui pratiquent la médiation au nom de la communauté qu'ils représentent $»^{51}$. Par conséquent, recourir au marché pour assurer la sécurité c'est ne pas en voir une des dimensions essentielles et ainsi ouvrir la voie à un certain nombre d'effets pernicieux.

Ainsi si Shearer présente l'intervention de EO en Angola (1993-1996) et en Sierra Leone (1995-1996) comme des succès évidents, ces exemples doivent au contraire nous faire réfléchir sur l'impact du recours à des entreprises de coercition. Il est vrai que EO, et ses quelques 500 personnes, ont réussi en Angola à contraindre l'UNITA à la table de négociation en 1995, même si son dirigeant, Jonas Savimbi ne signa jamais les accords de Lusaka. De la même façon, l'entreprise réussit à contraindre le RUF en Sierra Leone à signer un accord de paix en 1996 avec le gouvernement de Kabbah. Cependant, dans les deux cas le départ de EO fut l'une des conditions posées à la signature des accords. Ainsi ces conflits reprirent de plus belle après le départ de l'entreprise. Or, ce ne sont pas là des incidents fortuits liés au simple départ des entrepreneurs militaires comme le prétend Shearer. Ils trouvent leur origine dans leur intervention même. En effet, dans le cas de la Sierra Leone, le mécontentement des militaires suite à l'intervention de « mercenaires étrangers » créa des alliances objectives entre ceux-ci et le RUF. Ainsi en 1997, le gouvernement de Kabbah fut renversé par cette nouvelle alliance représentée par Koroma et le conflit repris. De la même façon, en Papouasie-Nouvelle-Guinée, l'intervention de Sandline International suscita une révolte de la part des militaires et le Premier ministre dut démissionner. Dans le cas de l'Angola, un accord imposé par une entreprise étrangère ne fut pas considéré par l'UNITA comme assez légitime pour être contraignant. Ainsi, le recours à des entreprises de coercition, si elle peut renforcer militairement un gouvernement, affaiblit souvent celui-ci en termes de légitimité politique, cela non seulement par rapport aux rebelles mais aussi par rapport aux forces gouvernementales elles-mêmes. En analysant les entreprises militaires selon 
des critères techniques, on se prive donc de la possibilité d'appréhender leurs effets politiques et structurels.

Cette critique a une portée beaucoup plus grande que celle selon laquelle les entrepreneurs militaires inscrivent leur action dans le court terme alors que la résolution de conflit suppose une perspective de long terme. En effet, elle ne remet pas seulement en cause la temporalité de l'intervention des entrepreneurs militaires, mais la nature même de l'acteur qui pose la régulation des conflits comme technique et non comme enjeu politique. De plus, elle va plus loin que les critiques traditionnelles qui se contentent de remettre en question les intentions de ces entreprises pour embrasser également la problématique des effets non-intentionnels, et pourtant potentiellement néfastes: il n'ait pas besoin de croire que les entreprises militaires sont intrinsèquement néo-coloniales ou des profiteurs de guerre pour remettre en cause leur capacité à jouer un rôle positif dans les conflits. Elle montre enfin que l'argument de l'efficacité, notamment militaire, passe à côté de l'essentiel.

La dimension politique du concept de sécurité a aussi une deuxième implication. En effet, en combinant une vision de la sécurité fondée sur la seule coercition, tout en s'inscrivant dans une logique de marché dans laquelle la solvabilité du client est le critère ultime, les entreprises de sécurité ne peuvent être productrices que d'exclusion. En effet, à la dimension exclusive de toute lecture sécuritaire conçue comme médiation entre menace et ordre, vient s'ajouter une logique de clientèle. Ainsi lorsque ces entreprises de sécurité se présentent comme fournisseurs de sécurité (security providers), la questionpertinente doit être "la sécurité de qui?». Alors que la marchandisation de la sécurité tend à banaliser celle-ci, il ne faut pas perdre de vue que la sécurité est, comme l'a montré Weaver, liée à des politiques d'exception qui ne s'appliquent pas de manière homogène dés lors qu'elles s'inscrivent dans une logique de clientèle. La sécurité devient ainsi un discriminant entre ceux qui ont les moyens de la payer et les autres. Lorsque de plus en plus d'individus et d'organisations se fient à l'industrie de la sécurité privée pour les rassurer et les protéger, il faut alors en mesurer les effets en termes d'enclavement et d'exclusion au sein de la société ${ }^{52}$. Le cas des gated communitiesaux Etats-Unis et en Afrique du Sud en fournit des exemples peu reluisants. Elles soulèvent de manière saillante la question du rapport entre sécurité et politique. Elles montrent aussi que la dimension « sécurité intérieure » des entreprises de coercition doit également être mise en évidence.

Les entreprises de coercition para-privées dans les champs des professionnels de la sécurité

En tant que professionnels de la sécurité se positionnant en tant que tels, les entrepreneurs de coercition répondent à un logique double mais inséparable de la concurrence économique d'une part et de la lutte d'intérêt au sein d'un champ des professionnels de la sécurité d'autre part. Ils sont affectés par les mêmes dynamiques que les acteurs publics de ce champ, à la différence qu'ils sont de surcroît contraints par des critères de rentabilité économique. Ainsi, ces entreprises partagent les systèmes de représentation et la doxa sur lesquels convergent les acteurs de ce champ, et participent aux luttes de définition et de hiérarchisation des menaces qui le caractérise. Il ne convient pas ici d'analyser dans le détail le rôle des acteurs privés au sein du champ de la sécurité, mais tout simplement de montrer que ces acteurs en subissent les effets, que ce soit au travers des services gouvernementaux qui les engagent ou de manière relationnelle, en même temps qu'ils participent à la reconfiguration de celui-ci. Comme l'a montré Didier Bigo, le champ des professionnels 
de la sécurité est caractérisé dans sa forme actuelle par une tendance à la dédifférentiation des questions de sécurité intérieure et de sécurité extérieure, tendance qui se nourrit notamment d'une extension de ce champ à de nouveaux enjeux ainsi sécurisés ${ }^{53}$.

Or, les entreprises de coercition militaire participent activement à cette tendance en se saisissant, de la même façon que les militaires ou parfois en tant que militaires, des thématiques relevant traditionnellement des opérations de police, voire de sécurité civile et donc de la sécurité intérieure. Cela est parfois facilité par d'anciens spécialistes de la sécurité intérieure (anti-terrorisme, lutte contre la drogue) qui se reconvertissent dans ces entreprises en même temps que des spécialistes de la sécurité extérieure. Ce positionnement au sein du champ de la sécurité s'explique par les querelles internes à ce champ sur ce qui constitue les nouvelles menaces à la sécurité et la manière idoine d'y répondre, mais aussi à une logique de diversification économique qui permet d'élargir le marché sur lequel ils opèrent. Ces deux logiques tendent d'ailleurs à se renforcer dans un contexte de flou quant à ce que constitue une menace à la sécurité. Ainsi, il n'est pas étonnant de voir certaines entreprises fournir de l'assistance médicale, lutter contre le trafic de drogue, faire du maintien de l'ordre, lutter contre le terrorisme, œuvrer dans le domaine de la consolidation de la paix, prétendre pouvoir gérer les risques environnementaux, faire de la prévention des conflits ou combattre la piraterie en Asie du Sud-Est. Northbridge Services Group s'est même récemment proposé de capturer l'ancien président libérien Charles Taylor pour qu'il soit traduit devant une instance juridictionnelle internationale. Ce faisant, ces entreprises contribuent aussi à la militarisation de ces pratiques en apportant leur savoir-faire spécifique fondé sur l'utilisation des technologies de surveillance et de contrôle de type militaire et une lecture exclusivement coercitive de la sécurité.

Cette militarisation de la gestion des peurs, à la fois permise et suscitée par les entrepreneurs militaires dans ce qui relevait traditionnellement de la sécurité intérieure, illustre bien que derrière le label sécuritaire c'est bien la question du rapport à la violence qui est posée. Commercialiser le label sécuritaire, c'est aussi transformer les pratiques coercitives dans le sens de la suppression d'un certain nombre de garde-fous caractéristiques de l'Etat de droit et notamment la distinction police/militaire. L'extension du marché de la sécurité rend la distinction entre marché national et marché international de plus en plus floue et renforce la tendance à la dédifférentiation du champ de la sécurité intérieure et de la sécurité extérieure. Ainsi, un certain nombre d'entreprises opèrent à la fois à l'échelle nationale et internationale en utilisant les mêmes types de technologies de coercition. Il s'établit ainsi un continuum technologique entre les opérations militaires et les opérations policières. Les effets de ce phénomène méritent d'être brièvement explicités.

L'entreprise DynCorps s'est illustrée en Colombie dans la lutte contre la drogue où elle a permis de gonfler les effectifs américains limités sur place, contribuant par-là à la militarisation de l'intervention. Ainsi, ne se contentant pas de piloter les avions chargés de détruire les champs de coca, elle aurait aussi participé dans ce cadre à la guerre secrète contre les guérillas locales ${ }^{54}$. Même s'il s'est agi d'une opération extérieure pour le compte du gouvernement américain, la nature de cette intervention, traditionnellement considérée comme policière et relevant notamment du DEA (Drug Enforcement Agency), témoigne du potentiel de militarisation du recours aux entreprises de coercition para-privées. Ce potentiel s'illustre plus particulièrement dans les activités de formation policière. Ainsi en 1996 des anciens soldats professionnels des 
SAS, embauchés par l'entreprise DSL, ont formé la police locale à la guerre contreinsurrectionnelle dans le cadre d'un contrat avec British Petroleum ${ }^{55}$.De la même façon, DynCorps a formé une partie de la police haïtienne. Or, dans les deux cas, ces opérations ont donné lieu à des entorses graves aux droits de l'homme: la police colombienne a fait l'objet de 169 rapports sur son implication dans des meurtres et des disparitions à la suite du contrat avec DSL et en Haïti la police s'est faite connaître pour ses violences gratuites et notamment des exécutions extra-judiciaires.

Il est difficile de connaitre avec exactitude l'implication du personnel de ces entreprises dans ces crimes. Néanmoins, la formation des polices par des anciens des forces spéciales (dans le cas de DSL) est peu susceptible de reproduire les modes de socialisation et les ethosspécifiques qui fondent traditionnellement la distinction entre polices et militaires. Comme l'a analysé Charles Moskos, l'ethos constabulaire des policiers (fondé sur le recours « minimal » à la force) se distingue traditionnellement de l'ethosmilitaire (fondé sur l'idée de l'utilisation de «toute la force nécessaire ») ${ }^{56}$. Il est vrai que notamment les opérations de consolidation de la paix ont contribué à brouiller cette distinction, mais le recours à des entreprises de coercition, embauchant des anciens militaires de carrière pour entrainer des polices en constitue certainement un des aspects les plus inquiétants. D'ailleurs, cette dé-différenciation ne concerne pas uniquement les modes de socialisation, mais aussi les systèmes technologiques auxquels. Ainsi, les employés de Sandline international qui sont intervenus sur l'île de Bougainville, des anciens des forces spéciales sud-africaines et britanniques, y ont été engagés avec des moyens militaires importants en tant que special constables ${ }^{57}$. Ce statut juridique spécial, relevant de la police, avait comme objectif de permettre à ces employés d'avoir recours directement à la force et à des arrestations, sans être soupçonnés d'activités mercenaires. Ici aussi on voit donc comment l'engagement d'entreprises militaires peut contribuer à la dé-différenciation entre sécurité intérieure et sécurité extérieure dans le sens d'une plus grande militarisation. Cela est d'autant plus le cas que, malgré les liens de ces entreprises avec les bureaucraties gouvernementales, le recours aux entreprises de coercition permet dans une certaine mesure d'autonomiser les professionnels de la sécurité de ceux de la politique (contournement $\mathrm{du}$ contrôle parlementaire et des individus réticents au sein des services gouvernementaux...) et donc établir d'avantage le champ de la sécurité comme champ de domination.

A la question de savoir «Qu'est-ce qui distinguait la violence exercée par les Etats de la violence exercée par n'importe qui d'autre?» au Moyen-Age, Tilly répond "sur le long terme suffisamment d'éléments pour rendre crédible la distinction entre force légitime et force "illégitime $»^{58}$. Or, ce qui est en jeu aujourd'hui au travers de l'émergence d'entreprises militaires n'est pas seulement la remise en question de cette frontière, mais aussi et surtout l'extension progressive de la sphère de la violence légitime (encore qu'il faudrait se demander: «légitime pour qui ?») au secteur para-privé. Or, cette extension pose problème dès lors qu'elle s'accompagne de surcroît d'une marchandisation du concept de sécurité et donc d'une redéfinition de la manière dont nous pensons le lien entre sécurité d'une part, politique et violence d'autre part. Cependant, sur le court terme c'est surtout la question de la limitation des effets potentiellement, mais aussi réellement, destructeurs de ces entreprises qui est posée. En effet, rien n'indique que les discours de légitimation utilisés en faveur de ces entreprises puissent être accrédités d'une quelconque crédibilité. Ils relèvent essentiellement $\mathrm{du}$ répertoire symbolique et semblent peu contraignants dans les 
pratiques sur le terrain. Pourtant la question reste ouverte. En effet, comme nous l'a montré cette analyse, la réponse ne se trouve probablement pas du côté des seules bureaucraties gouvernementales...

\section{NOTES}

1. . Etude réalisée par The Guardian citée dans « The Privatisation of war », Traynor I., The Guardian, mercredi 10 décembre, 2003.

2. . Ainsi dans Guerre et Contre-guerre (Fayard, Paris, 1994), les futurologues autoproclamés Alvin et Heidi Toffler affirment : «Quand les nations ont déjà perdu le monopole de la violence, pourquoi ne pas envisager de créer des forces de mercenaires volontaires organisées par des entreprises pour mener des guerres sur une base contractuelle pour le compte de l'ONU », (cité dans : de Saint-Quentin G., « Mercenariat et mutations stratégiques ", Défense Nationale, avril-juin 1998, p. 42).

3. . Voir notamment les rapports du rapporteur spécial de la Commission des Droits de l'Homme de l'ONU sur la question des mercenaires, M. Enrique Ballesteros : « L'Etat peut privatiser de nombreux secteurs de production et de services mais il ne peut pas privatiser ce qui est sa raison d'être » (Commission des Droits de l'Homme, Rapport sur la question de l'utilisation de mercenaires comme moyen de violer les droits de l'homme et d'empêcher l'exercice du droit des peuples de disposer d'eux-mêmes, 27 janvier 1998, p. 14). Ce positionnement se retrouve aussi dans un certain discours militaire notamment représenté par Grégoire de Saint-Quentin : « il paraît indispensable de redéfinir la place et le rôle du soldat, afin d'éviter que la violence, cessant d'être l'affaire des nations, devienne celle des acteurs sans légitimité » (SaintQuentin G., "Mercenariat et Mutations Stratégiques », Revue Défense Nationale, $\mathrm{n}^{\circ} 4$, avril-juin 1998, p. 44). Enfin, cette perspective semble aussi dans une certaine mesure structurer le point de vue de Anna Leander (voir Leander A., « Global Ungovernance : Mercenaries, States and the Control over Violence », www.copri.dk/publications/ workingpapers.htm).

4. . Voir van Creveld M., The Rise and Decline of the State, New York, Cambridge University Press, 1999.

5. . Sur cette distinction voir Badie B., L'Etat Importé, l'occidentalisation de l'ordre politique, Fayard, 1992.

6. . L'expression « entreprises de coercition para-privées » a été suggérée par Didier Bigo lors des travaux préliminaires à ce numéro.

7. . Voir les travaux de Brooks D., « Messiahs or Mercenaries? The future of international Private Military Services », International peace-keeping $n^{\circ} 7.4$, pp. 129-144 ; de Shearer D., Private Armies and Military Intervention, New York, IISS, Adelphi Paper 316, 1998 ; et de Leander A., « Global Ungovernance : Mercenaries, States and the Control over Violence " (www.copri.dk/publications/workingpapers.htm). 8. . Ainsi, depuis 1997, l'agence du renseignement militaire américain, la Defence intelligence Agency (DIA) organise des séminaires avec des représentants des grandes entreprises de sécurité, des représentants du Pentagone, des attachés militaires 
africains, des $\mathrm{ONG}$ ainsi que des agences onusiennes sur le thème de la privatisation de la sécurité.

9. . Edelman M., The Symbolic Use of Politics, Urbana, University of Illinois Press, 1964. 10. . Voir notamment : Brooks D., " Don't Kill the Cavalry, Rational Regulations for PMCs », papier présenté à la convention de l'International Studies Association (ISA), Portland (Oregon), 2003.

11. . Coker C., " Outsourcing War ", Cambridge Review of International Studies, vol. 12, $\mathrm{n}^{\circ} 1$, automne-hiver 1999, pp. 109 (traduction par l'auteur).

12. . On peut notamment évoquer les soupçons actuels à l'encontre de l'entreprise Kellog, Brown and Root (KBR), filiale de la firme Halliburton proche du camp des républicains, qui aurait massivement surfacturé des services rendus en Irak.

13. . Ainsi, MPRI disposerait d'un comité d'éthique et de contrôle de qualité.

14. 4. Macek L. « Mercenaires en ex-Yougoslavie dans les années 1990 », mémoire d'étudiant, IEP de Paris, 1999.

15. 5. Ces stratégies d'intégration sont particulièrement bien analysées dans : Banégas R., « De la guerre au maintien de la paix, le nouveau business mercenaire », Critique Internationale, $\mathrm{n}^{\circ} 1$, automne 1998, pp. 179-193.

16. 6. Voir à ce propos le compte-rendu des discussions relatives au procès de Sandline : Commission of Inquiry into Sandline, National Judicial Staff Services, Transcript of Proceedings (http://ww3.datec.com.pg/sandline/default.htm).

17. 7. Banégas $R$., « De la guerre au maintien de la paix, le nouveau business mercenaire », Critique Internationale, op. cit., p. 184.

18. 8. Ainsi, Shearer affirme : «ce rôle mixte n'est pas nécessairement un aspect négatif des activités d'une compagnie militaire : des gouvernements faibles ont eux-mêmes accueilli favorablement l'investissement étranger, particulièrement dans le secteur minier, rendu possible par les services de sécurité fournis par les entreprises privées. L'association d'intérêts économiques, de compagnies militaires et d'Etats est susceptible de se développer plus dans le futur pour devenir une composante importante de la stabilité interne de pays ayant une histoire de conflits internes » (traduction de l'auteur); Shearer D., « Private Military Force and Challenges for the Future ", Cambridge Review of international Affairs, vol. 12, n²1, automne-hiver 1999, p. 88.

19. . Spicer T., « Interview with Lt Col Tim Spicer », Cambridge Review of International Affairs, vol.12, n 1 automne-hiver 1999, pp. 165-171.

20. 0 . Voir à ce propos dans ce numéro : Kinsey $\mathrm{C}$., « Le droit international et le contrôle des mercenaires et des compagnies militaires privées ».

21. 1. Defaud N., « Mercenaires et pouvoir aux Comores, Concept 'd'île de la tortue' dans le système de représentation du mercenaire, micro-Etat et gestion étatique de la contrainte ", mémoire d'étudiant, IEP de Paris, 1999.

22. . Banégas R., « De la guerre au maintien de la paix, le nouveau business mercenaire ", op. cit., p. 185.

23. . Ainsi, en Sierra Leone, l'entreprise de gardiennage jadis rattachée à EO, Lifeguard, aurait en 1997 mené des opérations offensives contre les forces de la junte de Koroma en 1997 pour « sécuriser » des zones diamantifères dont pourtant la seule protection lui avait été confiée.

24. . Ainsi EO faisait partie d'un conglomérat plus vaste, la Strategic Ressources Corporation, de la même façon que Sandline aurait fait partie de Plaza 107 puis de The Deterrent Group. 
25. . Shearer D., Private Armies and Military Intervention, op. cit., p. 10.

26. . Shearer D., « Private Military Force and Challenges for the Future », op. cit., pp. 80-94.

27. . La Banque Mondiale aurait d'ailleurs directement négocié avec EO en 1995-1997 lors de l'opération de l'entreprise en Sierra Leone.

28. . Brooks D., « Don't Kill the Cavalry : Rational Regulation for PMCs », op. cit., p. 4.

29. . Ibid., p. 7.

30. . Silverstein K., « Privatising War », The Nation, 28 juillet-4 août, 1997, pp. 11-17.

31. . Chapleau P., Misser F., « Le retour des mercenaires », Politique Internationale, hiver 2001-2002, p. 236.

32. 2. Silverstein K., Private warriors, Londres, Verso, 2000, p. 172.

33. . Silverstein K., « Privatising War », The Nation, 28 juillet-4 août, 1997, p. 12.

34. O'Brien K., The role of External Security Forces in the Central African Crisis of 1997, Private armies and external instability (papier).

35. . Leander A., "Global Ungovernance, states and the control over violence », op. cit. 36. . O'Brien K., " Private Military Companies : Options for Regulation », memorandum (http://www.publications.parliament.uk).

37. . Strange S., The Retreat of the State : The Diffusion of Power in the World Economy, Cambridge, Cambridge University Press, 1996.

38. . Hibou B., « Retrait ou redéploiement de l'Etat ? ", Critique Internationale, $\mathrm{n}^{\circ} 1$, automne 1998, pp. 151-168.

39. 9. Rosenau, J. N., Turbulence in World Politics, Princeton, Princeton University Press, 1990.

40. . Wright-Mills C., L'élite du pouvoir, Paris, Maspéro, 1969.

41. . Polanyi K., La Grande Transformation, Aux origines politiques et économiques de notre temps, Paris, Gallimard, 1983.

42. . Waever O., "Securitisation and Desecuritisation ", in Lipschutz R. (ed.), On Security, New York, Columbia University Press, 1995, pp. 47-86.

43. 3. Je tiens ici compte d'une idée avancée par Didier Bigo.

44. 4. Leander A., « The Comodification of Violence, Private Military Companies and African States » (www.copri.dk/publications/workingpapers.htm).

45. 5. Bourdieu P., Ce que parler veut dire : l'économie des échanges linguistique, Paris, Fayard, 1982.

46. 6. Tilly C., « La guerre et la construction de l'Etat en tant que crime organisé ", Politix, vol. 13, n 49 (2000), p. 99.

47. 7. Shearer D., « Private Military Forces and Challenges for the Future », Cambridge Review of International Affairs, vol.12, n²1, automne-hiver 1999, p. 92.

48. 8. Voir notamment, Foucault M., Dits et écrits, t.IV, Gallimard 1994.

49. 9. Voir notamment Crowley J., « Pacifications et réconciliations : Quelques réflexions sur les transitions immorales », Cultures \& Conflits, $n^{\circ} 41$, printemps 2000 ; voir également son introduction.

50. 0. Lipshutz R., « On security » in Lipschutz R. (dir.), On Security, New York, Columbia University Press, 1995, pp. 1-23.

51. . Ceyhan Ayse, "Analyser la sécurité : Dillon, Waever, Williams et les autres ", Cultures \& Conflits, n³1-32, automne-hiver 1998, pp. 39-63.

52. 2. Voir dans ce numéro l'article de Pérouse de Montclos, M.-A., «Pétrôle et sécurité privée au Nigéria : un complexe multiforme à l'épreuve du 'syndrome de Monaco'». 
53. . Bigo D., « La Mondialisation de la sécurité ? Réflexion sur le champ des professionnels de la gestion des inquiétudes à l'échelle transatlantique et sur ses implications », article à paraître.

54. . Silverstein K., op. cit., 2000.

55. . Chapleau P., Misser F., op. cit., p. 234.

56. . Moskos C., « UN Peacemakers : the Constabulary Ethic and Military Professionalism », Armed Forces and Society, vol. 1, n4 1975, pp. 388-401.

57. 7. Voir « Agreement for the provision of military assistance dated this 31 day of january 1997 between the independent state of Papua New Guinea and Sandline International » (http://coombs.anu.edu.au/SpecialProj/PNG/htmls/Sandline.html). 58. . Tilly C., op. cit., p. 101.

INDEX

Mots-clés : entrepreunariat, Militaires, privatisation de la sécurité

Thèmes : Dyncorps, Executive Outcomes, MPRI 